up to birds; but there is much to interest anglers in those devoted to salmon- and trout-fishing. Mammals are in a minority; but perhaps the chapter describing caribou migration in Newfoundland is the most valuable in the book from the naturalist's point of view. A few chapters containing instructions and hints on bird and mammal photography, and on camping out, will be most helpful to those who wish to follow in Mr. Dugmore's steps and attempt to do what he has done under similar physical conditions.

"The Feet of the Furtive" (5) contains several well-written stories of a kind much in vogue at the present time, wherein the author weaves interesting facts in natural history into an attractive
Workers" (6), Mr. Claxton tells once again the story of the burying beetle, trapdoor spider, ants and aphides, wasps, and other common and familiar species of articulated animals the industries of which never fail to appeal to the imagination of children and to arouse their interest in creatures they are mostly taught by their elders to fear and destroy.

The purpose of awakening and fostering a taste for nature-study also underlies "Lessons from Nature's Workshop" (7), by the same author. This book, however, is rather more pretentious in scope than the last, and is written for readers of maturer mind, many of the chapters being devoted to more or less abstract questions in

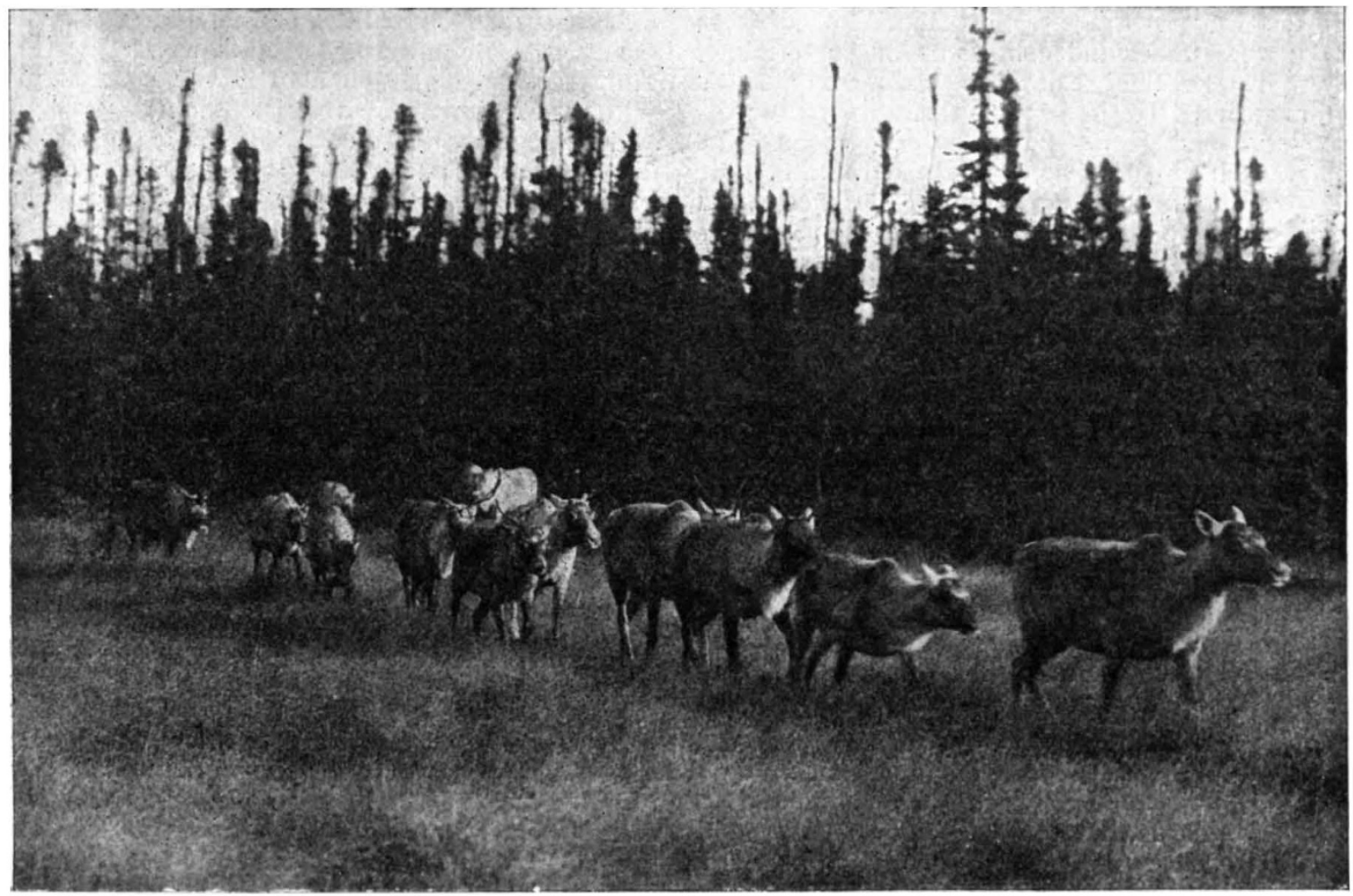

Fig. 2.-The Newfoundland caribou in migration. Going at a quick walk, or swinging trot, or at times a gallop, they usually travel in single file along the well-worn leads or paths that have been used for centuries. In nearly all cases a doe leads the herd. "From "Wild Life and the Camera."

fabric of fiction. The habits of familiar North American mammals are the theme Mr. Roberts presents so cleverly in this guise; but while giving full play to his imagination and to his powers of linguistic expression, he never oversteps the bounds of probability, and carefully avoids that pitfall authors too frequently dig for themselves and their readers, by attempting to humanise the species whose mode of life they wish to portray. Many of the stories recall others that have already been published by American authors; but there is a distinct air of novelty about the one called "The World of Ghost Lights," which gives a vivid picture of one aspect of life in the ocean depths.

In his little book for children, called "Insect natural history. such as the struggle for existence in plants, assimilative coloration, scenery, and so forth.

R. I. P.

\section{PROF. NOGUCHI'S RESEARCHES ON INFECTIVE DISEASES.}

THE Royal Society of Medicine mostly limits the record of its work to its own Proceedings and the medical journals; and it does well to observe this wise rule. But from time to time it receives some communication of the highest importance to the general welfare, and on such occasions it is mindful of its immediate duty to the public. It lately held a special meeting, at which Prof. Noguchi, of the Rockefeller Institute, demonstrated the results of his researches into NO. 2297 , VOL. $92 ?$ 
syphilis, general paralysis of the insane, epidemic infantile paralysis, and rabies. None who heard Prof. Noguchi and saw the great crowd of physicians and surgeons listening to him could fail to recognise the profound significance of this occasion.

No man of science works alone or in isolation : and a vast amount of cooperative work is being done in diverse parts of the world on what may be called the "higher types" of germs. Let us note the development of the work. Let us go back half a century, to the earliest methods of Pasteur. We may take 1855 as an approximate date for the beginning of the founding of "the germ-theory." For many years the only method which Pasteur had for the growth of germs in pure culture was the use of fluid media, such as broth; and, under the conditions of bacteriology fifty years ago, the use of these fluid media was full of difficulties. He had to wait until 1872 for the discovery that germs could be grown on solid media, such as gelatine or slices of potato. $\mathrm{He}$ had to wait until 1875 for the discovery that germs could be stained with aniline dyes so as to distinguish them, under the microscope, from their surroundings.

Pasteur lived until 1895 - that is, ten years after the first use of his protective treatment against rabies, and two years after the first use in practice of diphtheria antitoxin-but he did not live to see more than the beginning of the study of the higher types of germs. At the time when he died, many of the lower types-the bacilli and the micrococci -had been discovered, isolated, grown in pure culture on solid media, and proven, by the inoculation of test animals, to be the very cause of this or that infective disease. But the higher types, such as the plasmodium of malaria, were still waiting to be worked out. Then, after Pasteur's death, came Ross's fine work on malaria; and then came two discoveries of no less importance-the discovery (Schaudinn, Hoffmann) of Spirochaeta pallida in cases of syphilis, and the discovery (Forde, Dutton) of Trypanosoma gambiense in a case of sleeping sickness. These two discoveries brought syphilis and sleeping sickness, at last, within the range of practical bacteriology. Long ago, Moxon had said of syphilis that it was "a fever cooled and slowed by time"; but the cause of that fever was unknown until the Spirochaeta pallida was discovered.

But to prove that it does not merely accompany, but actually causes the disease, it had to be grown in pure culture, and inoculated into test animals, producing in them some characteristic sign. Syphilis must be studied as diphtheria, tetanus, typhoid fever, and tubercle had been studied. That is the meaning of all the work done by Ehrlich and his school upon salvarsan-that, in particles of tissue from a rabbit in which the disease has been produced, the Spirochaeta pallida is present, under the microscope, before a dose of salvarsan, and is absent after it.

The work has been of immeasurable complexity, and there is much still to be done. There are many species of spirochætes discoverable in this or that condition of bodily life, besides Spirochaeta pallida; indeed, Prof. Noguchi demonstrated seven species. But he has cleared the way in this field of bacteriology. He has distinguished those which need some air for their growth from those which cannot grow in air; he has discovered the method of adding a fragment of sterilised animal substance to each tube of pure culture: and these methods are of great value.

But that is not all. For he has detected Spirochaeta pallida in the brain, in general paralysis of the insane. He has found it in twelve out of seventy specimens. There is no need to underline the importance of that statement.

Also, Prof. Noguchi has obtained in pure culture the germs of anterior polio-myelitis (epidemic infantile paralysis). Of all the many diseases of childhood in which the art of medicine, apart from its science, is of no great use, few are more unkind than infantile paralysis. It is the Rockefeller Institute that we must thank here. First came Flexner's magnificent work on epidemic cerebrospinal meningitis, and his discovery (1908) of the special antitoxin for that disease; then came the study of epidemic infantile paralysis. To have in one's hands, in a test-tube, infantile paralysis, is a grand experience for a man who has attended a children's Hospital, year in year out, long before the Rockefeller Institute was born or thought of. It is enough to make him believe that the doctors some years hence may be able to stop the disease before it can inflict irremediable injury on the nerve cells of the spinal cord.

Finally, Prof. Noguchi spoke of rabies (hydrophobia). He has been able to obtain, in pure culture, the microscopic bodies which Negri discovered in the brain in that disease. He demonstrated to the Royal Society of Medicine, on the lantern-screen, photographs showing the cyclenot unlike that of the plasmodium malariaethrough which these bodies pass until, like miniature shrapnell, they break, setting free their constituent granules; and each granule becomes a "Negri body," and starts the cycle again. Happily, the protective treatment against rabies did not have to wait for the discovery of these Negri bodies. Pasteur worked at rabies, as Reed and Lazear worked at yellow fever, knowing that the virus was there, and able to control, fight, and beat it, without seeing it under the microscope.

The Royal Society of Medicine deserves the thanks of the public for inviting Prof. Noguchi to give this demonstration in London. He is indeed, in width and originality of work, equal to his fellow-countryman, Prof. Kitasato. He has helped to make it possible for men of science to extend to other diseases those methods of study which brought about the discovery of diphtheria antitoxin, and the protective treatments against cholera, typhoid fever, and plague.

\section{Stephen Paget.}

NO. 2297, VOL. 92] 